\title{
Produtividade de quatro espécies arbóreas de Terra Firme da Amazônia Central
}

\author{
Eleonora Alvarenga ANDRADE ${ }^{1}$, Niro HIGUCHI ${ }^{2}$
}

\begin{abstract}
RESUMO
A análise da produtividade individual de espécies permite descrever o comportamento das mesmas em ecossistemas florestais. O objetivo deste trabalho foi analisar a produtividade de quatro espécies arbóreas em um período de seis anos (2000-2006) em uma área de terra firme na região de Manaus, AM. A produtividade foi estimada indiretamente por meio da utilização de equações alométricas individuais geradas e dados de inventários florestais. Foram selecionadas Pouteria reticulata Eyma (Sapotaceae), Micrandra siphonioides Benth. (Euphorbiaceae), Protium hebetatum Daly (Burseraceae) e Eschweilera wachenheimii Sand (Lecythidaceae) por apresentarem alto índice de valor de importância na área. As equações foram geradas a partir de um arquivo de dados; devido ao reduzido número de indivíduos por espécie foi necessária a utilização de um método não paramétrico, sendo escolhido o "Jackknife". As equaçōes foram aplicadas às árvores das espécies selecionadas localizadas em duas parcelas permanentes com as informaçóes dos inventários florestais dos anos de 2000, 2002, 2004 e 2006. Os resultados mostram indícios de confiabilidade do método, as equações geradas apresentaram altos valores de coeficiente de determinação $\left(\mathrm{R}^{2}>0,93\right)$ e baixos valores de erro padrão da estimativa $\left(s_{y: x}<0,692\right)$, evidenciando sua consistência e precisão. As taxas de produtividade foram específicas para cada espécie, entretanto, o padrão de produtividade das espécies $E$. wachenheimii, $P$. reticulata e $P$. hebetatum foram semelhantes, diferindo da $M$. siphonioides. A maior produtividade se refere à espécie $M$. siphonioides e a menor à $P$. hebetatum. A variação da produtividade intra-específica foi superior à variação inter-específica, com P. reticulata apresentando o maior coeficiente de variação.
\end{abstract}

PALAVRAS-CHAVE: Floresta Densa de Terra Firme, Equações alométricas, Jackknife, Variação inter-específica, Variação intraespecífica.

\section{Productivity of four Terra Firme tree species of Central Amazonia}

\begin{abstract}
Individual productivity analysis of species helps describe their comportment in forest ecosystems. The objective of this work was to analyze the productivity of four tree species during a period of six years (2000-2006) in a "terra firme" area near Manaus, Amazonas. The productivity was indirectly estimated by using individual allometric equations along with data from a continuous forest inventory. The following species were selected for this study: Pouteria reticulata Eyma (Sapotaceae), Micrandra siphonioides Benth. (Euphorbiaceae), Protium hebetatum Daly (Burseraceae) and Eschweilera wachenheimii Sand (Lecythidaceae). These were selected because they presented a very high importance value index in the area. The raw data used to developed specific equations were taken from an existing set of data. Samples for each species numbered less than 30 individuals; for this reason, "Jackknife" was used. The "jackknifed" equations were used to estimate aboveground biomass of individuals which occurred in two permanent sample plots measured in 2000, and re-measured in 2002, 2004 and 2006. The "jackknife" method was consistent and reliable, with high and significant determination coefficients $\left(\mathrm{r}^{2}>0,93\right)$ and low values of standard error of estimates $\left(s_{y . x}<0,692\right)$. The productivity rates of $E$. wachenheimii, $P$. reticulata e P. hebetatum were similar, different from $M$. siphonioides. The highest productivity was reported for $M$. siphonioides, and the lowest for $P$. hebetatum. The intra-specific productivity variability was superior to the inter-specific, with $P$. reticulata showing the highest variability coefficient.
\end{abstract}

KEYWORDS: “Terra firme” dense forest, Allometric Equations, Jackknife, Inter-specific variability, Intra-specific variability

\footnotetext{
1 Professora Assistente da Universidade Federal do Amazonas, Instituto Agricultura e Ambiente. e-mail: ea_andrade@ufam.edu.br

2 Pesquisador do Instituto Nacional de Pesquisas da Amazônia. e-mail: niro@inpa.gov.br
} 


\section{INTRODUÇÃO}

A região amazônica é constituída por diferentes fitofisionomias, predominando a floresta de terra firme (Silva et al., 2004). Rizinni (1997) definiu esta fitofisionomia como floresta pluvial de grande porte localizada em planaltos pouco elevados $(60-200 \mathrm{~m})$, planos, ondulados ou recortada por cursos d'água não sujeito a inundaçôes, cujo substrato é de areia mais ou menos argilosa, amarelada ou avermelhada, podendo ser em poucos pontos, argiloso e fértil.

A produção primária líquida (PPL) representa o fluxo de carbono dentro do ecossistema; é definida como a diferença entre a fotossíntese total, que corresponde à produtividade primária bruta e a respiração autotrófica total (Clark et al., 2001). Na prática, no entanto, a PPL não pode ser definida em termos desta diferença (Clark et al., 2001). Uma definição alternativa é a quantidade líquida de carbono que é assimilado da atmosfera e fixado em nova matéria orgânica por unidade de tempo (DeLucia et al., 1999; Malhi et al., 2004).

Em ecossistemas terrestres seus componentes incluem produção de folhas, flores, frutos, raízes grossas e finas, tecidos lenhosos acima do solo (tronco e galhos), exportação de carboidratos para simbiontes e parasitas e formação de exsudatos e hidrocarbonetos voláteis. (Clark et al., 2001; Malhi et al., 2004).

A PPL global é estimada em $57 \mathrm{Gt}^{1}$ de carbono por ano e o estoque de carbono na vegetação corresponde a $640 \mathrm{Gt}$ (Field et al., 1998). As regiōes entre os trópicos apresentam as maiores taxas de produtividade da superfície terrestre, com destaque para as florestas tropicais que apresentam de 30\% a $50 \%$ da produtividade terrestre global e aproximadamente $40 \%$ do estoque terrestre global de carbono, sendo a maior parte alocada na forma de biomassa (Phillips et al., 1998; Grace et al., 2001).

A produtividade pode ser estimada por diferentes meios, tais como dados de sensoriamento remoto, modelos que utilizam parâmetros fisiológicos (índice de área foliar e fração absorvida da radiação fotossinteticamente ativa) e calculando o acúmulo de biomassa entre um ou mais anos (Gower, et al., 1999). A biomassa de uma área, por sua vez, pode ser estimada por meio de dois métodos: o método direto, que consiste na derrubada e pesagem das árvores que ocorrem em parcelas fixas e o indireto, que utiliza dados de inventários florestais e equações alométricas.

Alometria é o estudo das variações das formas e dos processos dos organismos. (Higuchi, et al., 2006). Equaçōes alométricas são modelos matemáticos oriundos de análise de regressão, são amplamente utilizadas como meio indireto de se estimar o volume madeireiro e a biomassa florestal de uma área (Higuchi \& Ramm, 1985; Weaver \& Gillespie, 1992).

$1 \quad 1 \mathrm{Gt}=1$ Gigatonelada $=10^{9}$ quilogramas
Estimar a biomassa de toda Amazônia pelo método direto é impossível, por se tratar de um método destrutivo, portanto, é preciso aperfeiçoar os métodos indiretos, onde as estimativas se baseiam em equaçôes alométricas para obter valores confiáveis e mais próximos do real no que se refere à biomassa e carbono acumulados em material vegetal (Higuchi et al., 2004).

Estudos acerca da produtividade em florestas tropicais são relevantes pelo fato de reterem grande parte do potencial mundial da produção primária e estoque de carbono. A dinâmica deste ecossistema pode influenciar as mudanças climáticas globais e composição atmosférica, tendo grande implicação econômica e impactos sobre a biodiversidade global.

Considerando as diferentes estratégias e o fato de que as espécies, e mesmo os indivíduos de uma mesma espécie, não se comportam de maneira semelhante torna-se necessária à avaliação do crescimento e produtividade individual.

Em uma área florestal manejada o conhecimento acerca da elasticidade da produtividade das espécies fornece suporte para decisōes sobre os tratamentos silviculturais a serem realizados. A prescrição de tratamentos adequados de acordo com a resposta de cada espécie proporciona uma otimização da atividade. Além disto, esta análise permite selecionar as espécies florestais que podem vir a ser exploradas e as que necessitam ser protegidas.

A pesquisa foi realizada com o intuito de estimar a produtividade de quatro espécies arbóreas de terra firme da Amazônia Central, bem como desenvolver equaçōes alométricas para estimar a biomassa aérea das espécies; correlacionar produtividade com classe de diâmetro e verificar se há variação de produtividade intra e interespecifica.

\section{MATERIAIS E MÉTODOS}

\section{COLETA DE DADOS}

O presente trabalho foi realizado na Estação Experimental de Silvicultura Tropical do Instituto Nacional de Pesquisas da Amazônia (EEST/INPA), núcleo ZF-2, distante cerca de $53 \mathrm{~km}$ em linha reta a noroeste de Manaus - Amazonas, coordenadas geográficas: $02^{\circ} 37^{\prime}$ a $02^{\circ} 38^{\prime}$ de latitude Sul e $60^{\circ} 09^{\prime}$ a $60^{\circ} 11^{\prime}$ de longitude Oeste, perfazendo 21.000 ha de área total.

A vegetação predominante é caracterizada como floresta tropical úmida densa de terra firme amazônica. O tipo climático é o "Amw" de acordo com a classificação de Köppen; caracterizado como tropical chuvoso com temperaturas elevadas (Ranzani, 1980).

A escolha das espécies foi baseada em um inventário florístico realizado por Carneiro (2004) em 7 dos 10 ha das transecçôes, área específica da pesquisa, descritas 
posteriormente. As espécies escolhidas foram Protium hebetatum, Pouteria reticulata, Micrandra siphonioides e Eschweilera wachenheimii, que estão entre as 20 espécies que apresentaram maior índice de valor de importância (IVI), devido ao elevado número de indivíduos. Atualmente, estas espécies não possuem valor comercial.

Inicialmente foram elaboradas equaçôes alométricas para estimar a biomassa da parte aérea das quatro espécies arbóreas escolhidas, geradas a partir de um modelo alométrico selecionado e um arquivo de dados. $\mathrm{O}$ referido arquivo pertence ao Laboratório de Manejo Florestal (LMF) do INPA. É composto por informaçôes do DAP, altura total e comercial e peso da parte aérea de 1117 árvores de diferentes espécies, cuja biomassa foi obtida utilizando o método direto, ou seja, o método destrutivo. Estas informaçóes foram coletadas na mesma estação experimental, EEST/INPA.

A partir deste, foram criados quatro arquivos para a realização do presente trabalho, cada um composto apenas por indivíduos de uma espécie. Sendo assim, foram utilizados dados de 11 indivíduos de E. wachenheimii, 12 de M. siphonioides, 9 de $P$. reticulata e 27 indivíduos de $P$. hebetatum.

$\mathrm{O}$ modelo alométrico selecionado foi:

$$
\ln \mathrm{p}=\mathrm{a}+\mathrm{b} \ln \mathrm{DAP}
$$

Sendo:

$\mathrm{P}=$ peso fresco (biomassa), em quilogramas;

$\mathrm{DAP}=$ diâmetro altura do peito, em centímetros;

$\ln =$ logaritmo natural;

$\mathrm{a}, \mathrm{b}=$ parâmetros a serem estimados.

A escolha do modelo foi baseada na literatura e na experiência do Laboratório de Manejo Florestal, considerando a simplicidade e praticidade. O modelo alométrico utilizado é de simples entrada, tendo como variável independente o DAP, que é de fácil medição. Equações alométricas para estimar a biomassa aérea gerada a partir deste modelo também foram propostas por West et al. (1997) apud Chambers et al. (2001), Chave et al., (2001) e Swamy et al. (2004).

A área específica, dentro da EEST/INPA, são duas transecções que medem $20 \mathrm{~m}$ de largura e $2500 \mathrm{~m}$ de comprimento com uma área de 5 ha cada e são estratificadas em platô, encosta e baixio. São parcelas permanentes onde todos os indivíduos com diâmetro altura do peito $(\mathrm{DAP}) \geq$ $10 \mathrm{~cm}$ estão marcados e identificados.

Nas transecçóes existem 31 indivíduos de Pouteria reticulata, 38 indivíduos de Micrandra siphonioides, 58 indivíduos de Protium hebetatum e 168 indivíduos de Eschweilera wachenheimii.

As equações individuais geradas foram aplicadas nos indivíduos das transecçôes com os dados dos inventários florestais dos anos de 2000, 2002, 2004 e 2006, para o cálculo da biomassa aérea. A produtividade foi estimada indiretamente pela biomassa acumulada entre duas mediçôes consecutivas. Todos os procedimentos referidos na coleta de dados foram processados utilizando-se o programa Microsoft Office Excel 2003.

\section{ANALISE DOS DADOS}

Devido ao reduzido número de indivíduos para a geração das equaçōes específicas por espécie, foi necessária a utilização de um teste não-paramétrico para a redução de tendências. No presente trabalho foi utilizado o método "Jackknife".

O método consiste em desdobrar os dados e recombinálos, re-utilizando a amostra diversas vezes, para estimar os parâmetros desconhecidos. Estima precisamente a variabilidade entre as estimativas computadas de todos os dados e estimativas quando cada grupo é removido. Estas diferenças geram valores que podem ser utilizados como se fossem oriundas de dados de distribuição normal (Higuchi, 1992).

Para comparar as médias dos parâmetros da produtividade das espécies ao longo do período analisado (2000-2006), foram realizadas análises de variância (ANOVA) de parcelas repetidas, onde os valores de $\mathrm{F}$ foram obtidos, porém a tomada de decisão foi feita de acordo com os coeficientes de correção de Greenhouse-Geisser Epsilon (G-G). Confirmada as diferenças pela ANOVA, testes post hoc de Tukey foram realizados, visando observar onde estas variaçôes eram significativas. Além disso, foram utilizadas correlaçôes lineares de Pearson (Pearson r), a fim de observar como as variáveis se relacionavam entre si.

Todas as diferenças a 5\% de probabilidade foram tidas como significantes, sendo que as análises estatísticas foram feitas utilizando-se o programa estatístico SYSTAT versão 10.2 para Windows.

\section{RESULTADOS E DISCUSSÃO}

\section{VARIAÇÃO INTER-ESPECÍFICA}

A partir do modelo escolhido, foi aplicado o método "Jackknife" para a geração das equações. Inicialmente foram calculados os coeficientes da equação, coeficiente de determinação $\left(R^{2}\right)$ e o erro padrão residual $\left(s_{v x}\right)$ por espécie em duas situações: quando todos os indivíduos foram processados e quando um subgrupo foi removido.

Posteriormente, para cada estimativa os valores foram transformados em pseudo valores por meio da fórmula descrita abaixo. $\mathrm{O}$ valor final para cada estimativa é a média dos pseudo valores. 
pseudo valores $=(r) \hat{\theta}-(r-1) \hat{\theta}_{-j}$

onde,

$\mathrm{r}$ = número de indivíduos da amostra

$\hat{\theta}=$ valor obtido quando são computados todos os indivíduos

$\hat{\theta}_{-j}=$ valor obtido quando um subgrupo é removido

Assim, as equações finais geradas para cada espécie foram:

\section{P. reticulata $-\ln$ peso $=-1,22+2,48 \ln \mathrm{DAP}$ \\ M. siphonioides $-\ln$ peso $=-1,10+2,41 \ln \mathrm{DAP}$ \\ $P$. hebetatum $-\ln$ peso $=-2,20+2,84 \ln \mathrm{DAP}$ \\ E. wachenheimii $-\ln$ peso $=-1,91+2,66 \ln \mathrm{DAP}$}

Todas estas equações apresentaram altos coeficientes de determinação $\left(\mathrm{R}^{2} \geq 0,93\right)$ e baixos valores de erro padrão residual $\left(s_{y: x} \leq 0,692\right)$, demonstrando que as equaçōes foram perfeitamente ajustadas pelo método e são consistentes e precisas.

Higuchi (1992) testou a eficiência do método "Jackknife" utilizando um banco de dados composto por 654 árvores e demonstrou que equações para estimar o volume, gerado a partir de apenas 10 árvores, produziram um erro de apenas $5 \%$ a mais do que quando foram utilizados todos os dados de um sub conjunto $(\mathrm{n}=327)$.

A fim de verificar a precisão das equaçôes geradas no presente trabalho, as mesmas foram aplicadas utilizando os mesmos dados que a geraram, assim como as equaçôes propostas por Higuchi et al. (1998):

$\ln$ peso $=1,754+2,665 \ln \mathrm{DAP}$; para DAP $\geq 20 \mathrm{~cm}$

$\ln$ peso $=-0,151+2,17 \ln \mathrm{DAP}$; para $5 \leq \mathrm{DAP}<20 \mathrm{~cm}$

Estes valores estimados por diferentes equaçôes foram comparados a media do peso real, ou seja, a média do peso de todos os indivíduos obtidos pelo método destrutivo, como demonstrado na Tabela 1 . Todos os valores estimados foram próximos ao peso real dos indivíduos; e considerando que as equaçōes de Higuchi et al. (1998) foram geradas a partir de dados de 315 árvores, estes resultados fornecem indícios da confiabilidade do método "Jackknife".

As taxas de produtividade das espécies nos intervalos de tempo analisados são mostradas na Tabela 2, onde também é possível observar o número de indivíduos presentes nos 10 ha.

A produtividade da espécie $M$. siphonioides foi superior a das demais espécies em todos os períodos, apesar do reduzido número de indivíduos e $P$. hebetatum apresentou
Tabela 1 - Média do peso real, da equação gerada no presente trabalho e das equações desenvolvidas por Higuchi et al. (1998) para as espécies: $P$. reticulata, M. siphonioides, $P$. hebetatum e $E$. wachenheimii.

\begin{tabular}{lllll}
\hline Espécie & $\begin{array}{l}N^{0} \\
\text { indivíduos }\end{array}$ & $\begin{array}{l}\text { Média do } \\
\text { peso real }\end{array}$ & $\begin{array}{l}\text { Média } \\
\text { equação } \\
\text { específica }\end{array}$ & $\begin{array}{l}\text { Média } \\
\text { equação Higuchi } \\
\text { et al. (1998) }\end{array}$ \\
\hline P. reticulata & 9 & 396,36 & 363,80 & 334,34 \\
M. siphonioides & 12 & 192,37 & 202,83 & 213,03 \\
\hline P. hebetatum & 27 & 136,94 & 147,72 & 136,47 \\
E. wachenheimii & 11 & 1166,31 & 996,19 & 1051,02 \\
\hline
\end{tabular}

a menor produtividade em dois dos três períodos. Não foram encontradas evidências claras de relacionamento entre o número de indivíduos e a produtividade média, sendo observadas fracas correlaçóes, tanto negativa para os intervalos de tempo 2000-2002 ( $r=-0,247 ; \mathrm{p}=0,752)$ e 2002-2004 ( $\mathrm{r}=-$ $0,311 ; \mathrm{p}=0,668)$, como positiva para o intervalo 2004-2006 $(\mathrm{r}=0,849 ; \mathrm{p}=0,151)$. Sendo assim, as taxas de produtividade podem ser consideradas independentes do número de indivíduos.

Finegan et al. (1999) sugeriram que espécies de crescimento rápido diferem das demais por apresentarem distribuição diamétrica irregular com relativamente poucos indivíduos nas classes de DAP inferiores, o que pôde ser verificado confrontando as distribuições diamétricas das espécies e as respectivas taxas de produtividade (Tabela 2, Figura 1). Podese, portanto, inferir que $M$. siphonioides seja uma espécie de crescimento rápido.

Pinto et al. (2003) reportaram que dos 23032 indivíduos encontrados em uma área de 18 ha no mesmo local de estudo, $83,0 \%$ possuíam DAP entre 5 e $20 \mathrm{~cm}$ e 17,0\% apresentavam $\mathrm{DAP}>20 \mathrm{~cm}$, sendo a maioria com padrão de distribuição com tendência ao agrupamento.

Ao se relacionar a produtividade média dos indivíduos das 4 espécies com o DAP, foram obtidas fortes evidências ( $\mathrm{p}$ $<0,001)$ de que há uma relação direta entre as variáveis e as espécies analisadas: $M$. siphonioides $(\mathrm{r}=0,592)$, E. wachenheimii $(\mathrm{r}=0,645)$, P. reticulata $(\mathrm{r}=0,715)$ e $P$. hebetatum $(\mathrm{r}=0,507)$. $\mathrm{E}$, considerando os diâmetros das árvores agrupados em três classes $(10 \leq \mathrm{DAP}<20 \mathrm{~cm} ; 20 \leq \mathrm{DAP}<30 \mathrm{~cm}$; DAP $\geq 30$ $\mathrm{cm}$ ), a produtividade periódica anual (IPA) da terceira classe foi significativamente superior $(\mathrm{p}<0,001)$ às demais, que se mostraram semelhantes entre si, como comprovado por estudos anteriores descritos abaixo.

As espécies $M$. siphonioides e $P$. reticulata apresentaram maior produtividade nas árvores com DAP superior a $40 \mathrm{~cm}$, sendo que $70,4 \%$ da produtividade da espécie $M$. siphonioides correspondem às árvores de diâmetro superior a $50 \mathrm{~cm}$. As espécies $E$. wachenheimii e $P$. hebetatum apresentaram maior produtividade nas classes de diâmetro inferiores (entre 10 e 30 $\mathrm{cm}$ ) (Tabela 3). Provavelmente este comportamento é reflexo da distribuição diamétrica dos indivíduos. 
Tabela 2 - Produtividade média das espécies nos intervalos de tempo e número de indivíduos analisados.

\begin{tabular}{lllll}
\hline $\begin{array}{l}\text { Produtividade } \\
\text { (kg/ha) }\end{array}$ & $\begin{array}{l}\text { Número de } \\
\text { indivíduos }\end{array}$ & 2000 - 2002 & 2002 - 2004 & 2004-2006 \\
\hline M. siphonioides & 39 & 1300,23 & 2170,33 & 1644,38 \\
\hline E. wachenheimii & 209 & 288,24 & 399,69 & 339,38 \\
P. reticulata & 33 & 70,93 & 146,77 & 121,19 \\
P. hebetatum & 65 & 94,97 & 111,50 & 76,74 \\
\hline
\end{tabular}

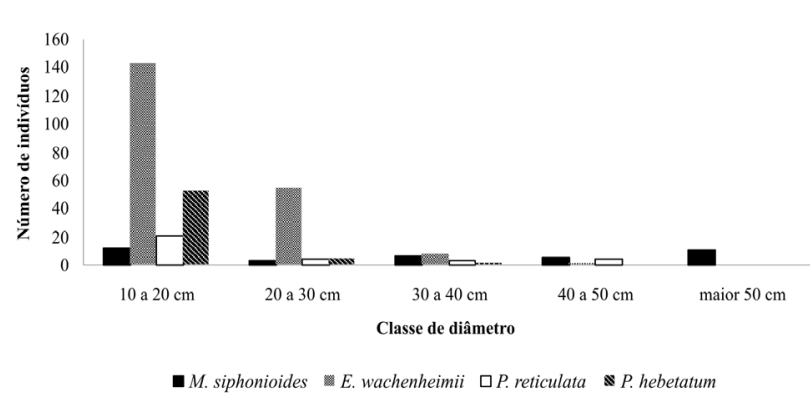

Figura 1 - Distribuição diamétrica dos indivíduos das espécies $M$. siphonioides, E. wachenheimii, $P$. reticulata e $P$. hebetatum.

M. siphonioides possui 16 indivíduos com DAP maior que $40 \mathrm{~cm}$, destes, 11 têm diâmetro superior a $50 \mathrm{~cm}$ e $P$. reticulata apresentou 4 indivíduos nas classes de diâmetro superiores. $P$. hebetatum não apresentou indivíduos nesta classe e $E$. wachenheimii apresentou apenas 1 indivíduo (Figura 1).

Silva (2001), afirmou que há diferença no crescimento entre as classes diamétricas, tendo verificado que árvores de maior porte diamétrico $(\mathrm{DAP} \geq 50 \mathrm{~cm})$ apresentam maiores taxas de crescimento. Clark e Clark (1996) verificaram maiores taxas de crescimento nas árvores com diâmetro entre 30 e 70 $\mathrm{cm}$ e as menores taxas encontravam nas classes de diâmetro inferiores a $30 \mathrm{~cm}$.

Hubbell et al. (1999) atribuem este maior crescimento em árvores maiores ao fato de serem árvores, na sua maioria, de dossel, com maior oferta de luz e conseqüentemente, maior taxa fotossintética e maior produção. King (2005) sugeriu que há diferença nas taxas de crescimento entre as espécies, devido não apenas as diferentes respostas fotossintéticas, mas também a atributos, tais como biomassa foliar por unidade de área, que afetam a interceptação luminosa.

Segundo Keller et al. (2001) em áreas de florestas tropicais as árvores com diâmetro superior a $35 \mathrm{~cm}$ retêm, aproximadamente, metade da biomassa total. Vieira (2003) estimou que, em uma área na região de Manaus, 70,8\% da biomassa encontra-se nas árvores pequenas e médias (DAP $<50 \mathrm{~cm}$ ), enquanto em Rio Branco (AC) e Santarém (PA) somente $58,6 \%$ e $45,1 \%$ da biomassa, respectivamente, estão nesta classe de diâmetro.
Tabela 3 - Percentagem da produtividade das espécies por classe de diâmetro.

\begin{tabular}{llllll}
\hline $\begin{array}{l}\text { Classe de diâmetro } \\
\text { (cm) }\end{array}$ & 10 a 20 & 20 a 30 & 30 a 40 & 40 a 50 & $<50$ \\
\hline M. siphonioides & $3,6 \%$ & $1,8 \%$ & $15,5 \%$ & $8,5 \%$ & $70,4 \%$ \\
\hline E. wachenheimii & $45,5 \%$ & $38,6 \%$ & $9,5 \%$ & $6,2 \%$ & $0 \%$ \\
\hline P. reticulata & $15,9 \%$ & $26,1 \%$ & $3,9 \%$ & $53,9 \%$ & $0 \%$ \\
\hline P. hebetatum & $75,1 \%$ & $22,0 \%$ & $2,8 \%$ & $0 \%$ & $0 \%$ \\
\hline
\end{tabular}

A análise de variância das taxas de produtividade das espécies indicou fortes evidências $(\mathrm{p}<0,001)$ da particularidade das mesmas, indicando que cada espécie se comportou de maneira distinta. Entretanto, o padrão de produtividade das espécies $E$. wachenheimii, $P$. reticulata e $P$. hebetatum foram semelhantes, diferindo da M. siphonioides (Figuras 2). Esta individualidade no crescimento das espécies já havia sido descrita por Clark \& Clark (2000) em áreas tropicais.

As quatro espécies apresentaram queda na produtividade no intervalo de tempo entre 2004 e 2006 (Tabela 2). Este declínio pode ser explicado por uma resposta a alguma adversidade ambiental, provavelmente uma distribuição anormal da precipitação ao longo do ano, como sugerido por Silva (2001). A autora encontrou fortes evidências na relação entre precipitação e crescimento, entretanto, sugere que o fator limitante ao crescimento não é a taxa de precipitação, mas a sua distribuição ao longo do ano.

De acordo com os dados de precipitação anual do Laboratório de Manejo Florestal coletados na área de estudo (Tabela 4) não houve grande redução na precipitação anual neste período, entretanto, a sua distribuição no ano de 2005 foi anormal, comparado à anos anteriores.

Miranda (2002) não verificou correlação significante entre crescimento e precipitação, apesar de um maior crescimento estar associado à estação chuvosa com um maior índice pluviométrico. Vieira (2003) encontrou esta sazonalidade no crescimento, sendo mais pronunciado nas árvores com diâmetro acima de $50 \mathrm{~cm}$.

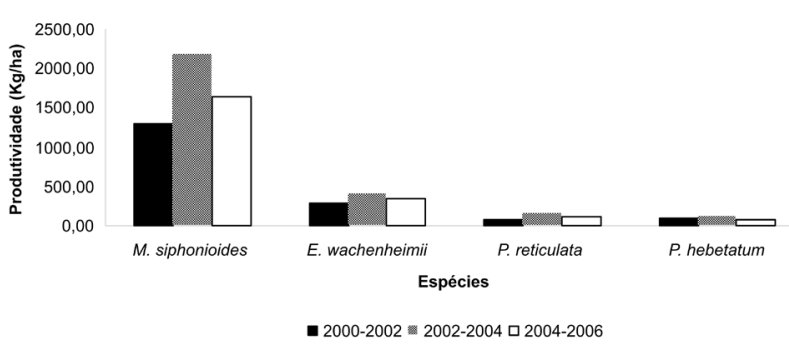

Figura 2 - Padrão de produtividade das espécies $M$. siphonioides, $E$. wachenheimii, $P$. reticulata e $P$. hebetatum. 
Tabela 4 - Precipitação anual referente aos anos de 2000 a 2006.

\begin{tabular}{ll}
\hline Anos & Precipitação Anual $(\mathrm{mm})$ \\
\hline 2000 & 3565,9 \\
2001 & 2356,7 \\
2002 & 3177,7 \\
2003 & 2044,2 \\
2004 & 2811,8 \\
2005 & 2698,2 \\
2006 (jan-jun) & 1823,6 \\
\hline
\end{tabular}

Outros fatores que influenciam na produtividade são as taxas de mortalidade e recrutamento (quando uma árvore alcança um DAP mínimo de $10 \mathrm{~cm}$ e ingressa no inventário). Um balanço entre ingresso e mortalidade determina se uma comunidade está aumentando ou diminuindo com o tempo. O incremento no DAP das árvores, juntamente com o recrutamento contribui positivamente, enquanto a mortalidade contribui de forma negativa (Poels et al. 19980 apud Rocha et al. 2003). Estes processos ecológicos apresentam alta variação anual e inter-específica (Scheller \& Mladenoff, 2004).

Rocha et al. (2003) sugeriram que em períodos de estiagens prolongadas, como acontece na Amazônia em anos que ocorrem o evento climático El Niño, a mortalidade das árvores aumenta, porém a maioria delas permanece em pé e vão se decompondo gradualmente com o passar do tempo.

No período analisado houve taxa de recrutamento em todos os inventários: 2002 (5,1\%), 2004 (0,3\%) e 2006 $(4,4 \%)$, sendo que a espécie $E$. wachenheimii contribuiu com $2,1 \%, 0,3 \%$ e $1,7 \%$; P. hebetatum contribuiu com $1,8 \%, 0 \%$ e $1,5 \% ; M$. siphonioides contribuiu com $0,9 \%, 0 \%$ e $0,3 \%$ e $P$. reticulata contribuiu com $0,3 \%, 0 \%$ e $0,9 \%$ respectivamente para os valores acima citados. Taxa de mortalidade foi verificada apenas em 2006 (4,7\%), com a maior taxa referente à espécie $P$. hebetatum (2,6\%), seguida por $M$. siphonioides e $E$. wachenheimii com $0,9 \%$ cada e $P$. reticulata com $0,3 \%$.

Apesar de P. reticulata e E. wachenheimii terem apresentado taxas de recrutamento superiores às de mortalidade em 2006, as taxas de produtividade apresentaram queda comparadas ao período anterior.

As espécies $P$. hebetatum e $M$. siphonioides apresentaram taxas de mortalidade superiores às de recrutamento em 2006 $\mathrm{e}$ as taxas de produtividade acompanharam esta tendência, ou seja, foram inferiores as produtividades do período anterior.

O comportamento das espécies $P$. reticulata e $E$. wachenheimii pode ser explicado pela perda de indivíduos com grandes DAPs, que como discutido anteriormente, apresentam elevada produtividade, o que não foi compensado pelo ingresso de indivíduos com baixos DAPs. Da mesma forma, a perda de indivíduos grossos afetou a produtividade das espécies $P$. hebetatum e M. siphonioides.

No período entre 1996 e 2000, Rocha et al. (2003) determinaram a taxa de recrutamento $(0,9 \%)$ e mortalidade $(0,9 \%)$ para uma área no mesma local de estudo. Dentre as 11 espécies que mais contribuíram, a taxa de recrutamento variou entre 13,4\% (Protium sp.) e 1,8\% (Eugenia sp.) e a taxa de mortalidade entre $11,1 \%$ e 2,8\% referentes às espécies acima citadas.

\section{VARIAÇÃO INTRA-ESPECÍFICA}

$A$ variação da produtividade entre os indivíduos foi altamente significativa $(\mathrm{p}<0,01)$, sendo superior à variação entre as espécies. Todas as espécies apresentaram elevado coeficiente de variação $(\mathrm{CV})$ entre os indivíduos. P. reticulata apresentou o maior CV (157,7\%), seguida por M. siphonioides $(144,7 \%)$, E. wachenheimii $(121,0 \%)$ e $P$. hebetatum $(98,3 \%)$.

Os altos CV indicam que as espécies apresentam alguma elasticidade, ou seja, o bom crescimento depende de condições favoráveis, que pode ser posição topográfica, associação botânica, dentre outros. Além disto, se aplicados tratamentos silviculturais devem responder positivamente a estes.

Silva (2001) encontrou uma variação grande entre indivíduos na mesma área de estudo, analisando, entretanto, a variação em diâmetro por meio de bandas dendrométricas. A autora relatou que o CV de 11 espécies analisadas variou de $38 \%$ a $431 \%$.

Miranda (2002) comprovou a existência de padrões e taxas de crescimento diferenciadas entre indivíduos de uma mesma espécie, verificando a resposta individual e característica. Segundo a autora, este comportamento pode ser explicado pela idade dos indivíduos, vigor metabólico, estado fitossanitário, heterogeneidade do solo, estrutura do dossel e competição ou associação entre espécies e indivíduos.

Higuchi et al. (2003) acrescentaram as variações de estaçôes do ano e condições microclimáticas e afirmaram que para inferir sobre o padrão de crescimento individual em diâmetro de árvores da floresta amazônica, diversos outros fatores exercem influência e precisam ser considerados. Dentre esses fatores, o autor mencionou a grande heterogeneidade de espécies e ecossistemas, as características peculiares de cada espécie, fenologia dos grupos ecológicos associadas aos fatores ambientais bióticos e abióticos e as características climatológicas de cada região.

\section{CONCLUSÕES}

O método Jackknife, é um teste não paramétrico de redução de tendências de dados de distribuição livre. Por 
meio de sua aplicação foi possível gerar precisas equações alométricas específicas a partir de um número reduzido de dados, o que possibilitou avaliar a produtividade das espécies na área selecionada.

As equações geradas apresentaram altos valores de coeficiente de determinação $\left(\mathrm{R}^{2}>0,93\right)$ e baixos valores de erro padrão $\left(s_{y, x}<0,692\right)$. O peso por elas estimado foi próximo ao peso real, ou seja, obtido pelo método destrutivo. Sendo assim, o método, que vem sendo amplamente utilizado em diversas áreas, se mostrou útil e confiável em análises desta natureza.

As taxas de produtividade foram específicas para cada espécie, demonstrando que elas se comportam de maneira distinta e respondem diferentemente às condiçōes ambientais. Contudo, o padrão de produtividade das espécies $E$. wachenheimii, $P$. reticulata e $P$. hebetatum foram semelhantes, diferindo da M. siphonioides, que se destacou pela elevada produtividade ao longo de todo período analisado.

Em todas as espécies foi encontrada uma relação direta entre produtividade e diâmetro das árvores, ou seja, houve diferença na produtividade entre as classes diamétricas. Este comportamento foi mais pronunciado nos indivíduos com diâmetro superior a $30 \mathrm{~cm}$. Esta constatação pode explicar, em parte, a elevada produtividade de M. siphonioides, pois esta espécie possuía maior número de indivíduos de grande porte.

A variação da produtividade intra-específica foi altamente significativa, sendo superior à variação inter-específica. Todas as espécies apresentaram elasticidade, com elevados coeficientes de variação. Dentre as quatro espécies, a produtividade de $P$. hebetatum foi mais homogênea $(\mathrm{CV}=98,3 \%)$ e $P$. reticulata apresentou a maior variação $(\mathrm{CV}=157,7 \%)$.

\section{BIBLIOGRAFIA CITADA}

Carneiro, V.M.C. 2004. Composição florística e análise estrutural da floresta primária de terra firme na bacia do rio Cueiras, Manaus $A M$. Dissertação de mestrado, Instituto Nacional de Pesquisas da Amazônia, Manaus, Amazonas. 77pp.

Chambers, J.Q., Santos, J. dos, Ribeiro, R.J., Higuchi, N. 2001. Tree damage, allometric relationships and above-ground in central Amazon forest. Forest Ecology and Managements, 152: 73-84.

Chave, J.; Riéra, B.; Dubois, M. 2001. Estimation of biomass in a neotropical forest of French Guiana: spatial and temporal variability. Journal of Tropical Ecology, 17: 79-96.

Clark, D.A.; Brown, S.; Kicklighter, D.W.; Chambers, J.Q.; Thomlinson, J.R.; Ni, J. 2001. Measuring net primary production in forest: concepts field methods. Ecological Applications, 11(2): 356-370.

Clark, D.B.; Clark, D.A. 2000. Landscape-scale variation in forest structure and biomass in a tropical rain forest. Forest Ecology and Management, 137: 185-198.
Clark, D.B.; Clark, D.A. 1996. Abundance, growth and mortality of very large trees in neotropical lowland rain forest. Forest Ecology and Management, 80: 235-244.

DeLucia, E.H.; Hamilton, J.G.; Naidu S.L.; Thomas, R.B.; Andrews J.A.; Finzi, A.; Lavine, M.; Matamala, R.; Mohan, J.E.; Hedrey, G.R.; Schlesinger, W.H. 1999. Net primary production of a forest ecosystem with experimental $\mathrm{CO}_{2}$ enrichment. Science, 284: 1177-1179.

Field, C.B.; Behrenfeld, M.J.; Randerson, J.T.; Falkowski, P. 1998. Primary production of the biosphere: integrating terrestrial and oceanic components. Science, 281: 237-240.

Finegan, B.; Camacho, M.; Zamora, N. 1999. Diameter increment patterns among 106 tree species in a logged and silviculturally treated Costa Rican rain forest. Forest Ecology and Management, 121: $159-176$

Gower, S.T.; Kucharik, C.J.; Norman, J.M. 1999. Direct and indirect estimation of leaf area index, $\mathrm{fAPAR}$, and Net Primary Production of terrestrial ecosystem. Remote Sens. Environ, 70: 29-51.

Grace, J.; Malhi, Y.; Higuchi, N.; Meir, P. 2001. Productivity of tropical rain forest. In: Roy, J.; Saugier, B.; Mooney, H.A. (Eds.) Terrestrial global productivity. Academic Press London, England. p. 401-426.

Higuchi, N.; Lima, A.J.N.; Teixeira, L.M. 2006. Apostila Biometria Florestal. Instituto Nacional de Pesquisas da Amazonia, Manaus, Amazonas. 140pp.

Higuchi, N.; Chambers, J.; Santos, J. dos; Ribeiro, R.J.; Pinto, A.C.M.; Silva; R.P. da; Rocha, R.M.; Tribuzy, E.S. 2004. Dinâmica e balanço do carbono da vegetação primária da Amazônia Central. Floresta, 34(3): 295-304.

Higuchi, N.; Chambers, J.Q.; Silva, R.P. da; Miranda, E.V. de; Santos, J. dos; Iida, S.; Pinto, A.C.M.; Rocha, R.M.; Souza, C.A.S. de. 2003. Uso de bandas metálicas e dendrômetros automáticos para a definição do padrão de crescimento individual das principais espécies arbóreas da floresta primária da região de Manaus, Amazonas, Brasil. In: Higuchi, N.; Santos, J. dos; Sampaio, P.T.B.; Marenco, R.A.; Ferraz, J.; Sales, P.C. de; Saito, M.; Matsumoto, S. 2003. Projeto Jacarandá Fase II: Pesquisas Florestais na Amazônia Central. Instituto Nacional de Pesquisas da Amazonia, Manaus, Amazonas. p. 55-68.

Higuchi, N.; Santos, J. dos; Ribeiro, R.J.; Minette, L.; Biot, Y. 1998. Biomassa da parte aérea da vegetação da floresta tropical úmida de terra-firme da Amazônia brasileira. Acta Amazonica, 28(3): 153-165.

Higuchi, N. 1992. Usando o método "Jackknife" para estimar volume de madeira da Floresta Amazônica. Atas da 24a Reunião regional da associação brasileira de estatística, $12^{a}$ Semana do estatístico. Manaus. p. 42-56.

Higuchi, N.; Ramm, W. 1985. Developing bole wood volume equations for a group of tree species of Central Amazon (Brazil). Commonwealth Forestry Review, 64(1): 33-41.

Hubbell, S.P.; Foster, R.B.; O`Brien, S.T.; Harms, K.E.; Condit, R.; Wechsler, B.; Wright, S.J.; Lao, S.L. 1999. Light-gap disturbances, recruitment limitation, and tree diversity in a Neotropical Forest. Science, 283: 554-557. 
Keller, M.; Palance, M.; Hurtt, G. 2001. Biomass estimation in the Tapajós National Forest, Brazil. Examination of sampling and allometric uncertainties. Forest Ecology and Management, 154(3): 371-382.

King, D.A. 2005. Linking tree form, allocation and growth with an allometrically explicit model. Ecological Modelling, 185: 77-91.

Malhi, Y.; Baker, T.R.; Phillips, O.L.; Almeida, S.; Alvarez, E.; Arroyo, L.; Chave, J.; Czimczik, C.I.; Di Fiore, A.; Higuchi, N.; Killeen, T.J.; Laurance, S.G.; Laurance, W.F.; Lewis, S.L.; Montoya, L.M.M.; Monteagudo, A.; Neill, D.A.; Vargas, P.; Patiño, S.; Pitman, N.C.A.; Quesada, C.A.; Salomão, R.; Silva, J.N.M.; Lezama, A.T.; Martínez, R.V.; Terborgh, J.; Vinceti, B.; Lloyd, J. 2004. The above-ground coarse wood productivity of 104 neotropical forest plots. Global Change Biology, 10(5): 563-591.

Miranda, E.V.de. 2002. Padrão de desenvolvimento radial horário do fuste de três espécies florestais típicas da Amazônia utilizando dendrômetros automáticos. Dissertação de mestrado, Instituto Nacional de Pesquisas da Amazônia, Manaus, Amazonas. $75 \mathrm{pp}$.

Philips, O.L.; Malhi, Y.; Higuchi, N.; Laurence, W.F.; Nunez, P.V.; Vasquez, R.M.; Laurence, S.G.; Ferreira, L.V.; Stern, M.; Brown, S.; Grace, J. 1998. Change in the carbon balance of tropical forest: evidence from long-term plots. Science, 282: 439-442.

Pinto, A.C.M.; Higuchi, N.; Iida, S.; Santos, J. dos; Ribeiro, R.J.; Rocha, R.M.; Silva, R.P.da. 2003. Padrão de distribuição espacial de espécies florestais que ocorrem na região de Manaus-AM. In: Higuchi, N.; Santos, J. dos; Sampaio, P.T.B.; Marenco, R.A.; Ferraz, J.; Sales, P.C. de; Saito, M.; Matsumoto, S. 2003. Projeto Jacarandá Fase II: Pesquisas Florestais na Amazônia Central. Instituto Nacional de Pesquisas da Amazonia, Manaus, Amazonas. p. 1-20.

Ranzani, G. 1980. Identificação e caracterização de alguns solos da Estação Experimental de Silvicultura Tropical do INPA. Acta Amazonica, 10(1): 7-41.

Rizinni, C.T. 1997. Tratado de fitogeografia do Brasil: aspectos ecológicos, sociológicos e florísticos. $2^{\mathrm{a}}$ ed. Âmbito Cultural ediçōes Ltda, Sao Paulo, Sao Paulo. 747p.
Rocha, R.M.; Higuchi, N.; Santos, J. dos; Nakamura, S.; Silva, R. da; Pinto, A.C.M.; Tribuzzy, E.S. 2003. Taxas de recrutamento e mortalidade e mudanças de estoques de fitomassa da floresta primária na região de Manaus-AM. In: Higuchi, N.; Santos, J. dos; Sampaio, P.T.B.; Marenco, R.A.; Ferraz, J.; Sales, P.C. de; Saito, M.; Matsumoto, S. 2003. Projeto Jacarandá Fase II: Pesquisas Florestais na Amazônia Central. Instituto Nacional de Pesquisas da Amazonia, Manaus, Amazonas. p. 43-54.

Scheller, R.M.; Mladenoff, D.J. 2004. A forest growth and biomass module for a landscape simulation model, LANDIS: design, validation and application. Ecological Modelling, 180: 211229.

Silva, N.M.C. da; Antony, L.M.K.; Rocha, R.M.; Silva, R.P.S.; Carneiro, V.M.; Teixeira, L.M.; Veiga, J.V.; Higuchi, N. 2004. A biosfera: seus componentes e conceitos In: Higuchi, M.I.G.; Higuchi, N. 2004. A Floresta amazônica e suas múltiplas dimensôes: uma proposta de educação ambiental. Instituto Nacional de Pesquisas da Amazonia, Manaus, Amazonas. p. 17-44.

Silva, R.P. 2001. Padrōes de crescimento de árvores que ocorrem em diferentes toposseqüências na regiāo de Manaus-AM. Dissertação de mestrado, Instituto Nacional de Pesquisas da Amazônia, Manaus, Amazonas. 57pp.

Swamy, S.L.; Kushwaha, S.K.; Puri, S. 2004. Tree growth, biomass, allometry and nutrient distribution in Gmelina arborea stands grown in red lateritic soils of Central India. Biomass and Bioenergy, 26: 305-317.

Weaver, P.L.; Gillespie, A.J.R. 1992. Tree biomass equations for the forests of the Luquillo Mountains, Puerto Rico. Commonwealth Forestry Review, 71(1): 35-39.

Vieira, S.A. 2003. Mudanças globais e taxa de crescimento arbóreo na Amazônia. Tese doutorado, Escola Superior de Agricultura Luiz de Queiroz, Piracicaba, São Paulo. 103pp.

Recebido em 13/02/2008

Aceito em 26/06/2008 